\title{
Evaluation der Arbeitsmarktpolitik - Ein Schritt vor und zwei zurück?
}

Wann hat es das zum letzten Mal gegeben? Die Bundesagentur für Arbeit (BA) sorgt für positive Schlagzeilen. Mit dem unerwartet starken Konjunkturaufschwung darf sie endlich steigende Beschäftigung und sinkende Arbeitslosigkeit melden. Auch der Finanzminister kann sich freuen. Die Presse verkündet: Die BA hat im Jahr 2006 11,2 Mrd. € „erwirtschaftet" - so als ob die BA ein Profitcenter der Bundesregierung wäre. Doch obwohl im Jahresdurchschnitt 2006 noch 4,5 Mio. Arbeitslose registriert waren, blieben mehr als $15 \%$ der zur Verfügung stehenden Mittel für Arbeitsförderung und Eingliederung von Arbeitslosen ungenutzt. Was erklärt dieses Paradox?

Die Evaluation der Arbeitsmarktpolitik trug ein gerüttelt Maß dazu bei. Die Evaluationsforschung erster Stufe (Anfang der 1990er Jahre) beschränkte sich weitgehend auf Bruttoeffekte (Teilnehmerzahlen, Verbleibs- und Integrationsquoten) und löste mit Blick auf einige Instrumente die Illusion hoher Wirksamkeit aus. Die Desillusion lieferte die Evaluationsforschung zweiter Stufe (Ende der 1990er Jahre), die mit strengen Kontrollgruppenverfahren, aber kurzfristigen Zeithorizonten überwiegend negative Effekte konstatierte. Die jüngsten Reformen lösten die Evaluationsforschung dritter Stufe aus. Eine wachsende Zahl professioneller Forscher kann nun auf der Basis einer modernen Dateninfrastruktur rechnen, quantitative mit qualitativen Verfahren verbinden und längerfristige Wirkungshorizonte berücksichtigen.

So notwendig wie erfolgreich die Entwicklung dieser Evaluationskultur war, so ambivalent ist die Umsetzung ihrer Ergebnisse in der Politik: Je robuster die Evaluationsmethoden, desto leichter scheint es, Maßnahmen durch Berufung auf negative Evaluationsergebnisse zurückzufahren, ohne gleichzeitig im entsprechenden Umfang auf effiziente Instrumente umzuschwenken. Die frühere Evaluationsforschung gab jedenfalls der Auffassung reichlich Nahrung, Arbeitsförderung zahle sich nicht aus. Die Meinung ist weit verbreitet, der Markt werde es schon richten, und wo nicht, müsse mit weiteren Kürzungen von Leistungen oder „Aktivierung“ nachgeholfen werden. Dagegen liefert die jüngere Evaluationsforschung alles andere als Gründe, in arbeitsmarktpolitische Abstinenz zu flüchten. Ihre Ergebnisse zeichnen zwar ein insgesamt differenziertes Bild, für einige wichtige Instrumente sind aber durchaus Erfolge erkennbar. Die Reform der Arbeitsvermittlung ist auf gutem Wege, wie mehrere - schon in einem frühen Stadium von der Hans-BöcklerStiftung geförderte - Studien des WZB zeigen. Die berufliche Weiterbildung und Eingliederungszuschüsse erhöhen ebenso wie Existenzgründungen die Arbeitsmarktchancen. Der Vergleich von Evaluationsstudien vor und nach den Reformen (also seit 2002) macht auch deutlich, dass mit dem Reformprozess die Wirksamkeit einzelner Maßnahmen (insbesondere der Weiterbildung) gesteigert werden konnte. Ebenso erfreulich ist, dass mit der Evaluationsforschung dritter Stufe eine klarere Trennung zwischen wirksamen und schäd- lichen Instrumenten vorgenommen werden kann. Dass Politik auf dieser Basis auch zu lernen bereit ist, zeigt die veränderte Stoßrichtung der Ausgaben. Nach Berechnungen des Instituts zur Zukunft der Arbeit (IZA) können nun etwa $28 \%$ der Ausgaben als wirksam eingeschätzt werden, während der Anteil negativ evaluierter Maßnahmen auf $1,5 \%$ zurückging.

Evaluationsforschung und Arbeitsmarktpolitik haben so einen klaren Schritt voran gemacht. Das reicht jedoch nicht aus, und es drohen Rückschritte. Einen solchen hat die Politik schon vollzogen: Obwohl die jüngsten Evaluationen insbesondere der Weiterbildung positive Effekte bescheinigen und obwohl die Fachkräftelücke immer größer wird, sind die Teilnehmerzahlen in Weiterbildungsmaßnahmen von 500.000 im Jahre 2000 auf 132.000 im Jahre 2005 zurückgegangen. Ferner ist die einschränkende Reform der Existenzgründungsförderung - insbesondere deren Begrenzung auf Arbeitslosengeldempfänger - ebenso fragwürdig wie die im Gegenzug explosive Förderung kurzfristiger Trainingsmaßnahmen weitgehend perspektivloser Arbeitsgelegenheiten („Ein-Euro-Jobs“) und Minijobs. Auch die Evaluationsforschung droht auf halbem Wege stehen zu bleiben: Bisher ist nur knapp ein Drittel der derzeitigen Maßnahmen durch die Evaluationsforschung erfasst. Wichtige Bereiche der Arbeitsförderung (Jugendliche, Behinderte, der gesamte SGB II-Bereich, Altersteilzeit) werden erst noch evaluiert oder sind noch gar nicht zur Evaluation vorgesehen. Weitere Schritte sind notwendig, um die Evaluationsforschung auf die vierte Stufe zu heben: Die Evaluationskultur muss nun von ihrer ad hoc-Struktur auf eine kontinuierliche Basis gestellt werden; methodische Fortschritte im Kontrollgruppendesign oder in der experimentellen Forschung sind dringend erforderlich; die Langfrist- und Nebenwirkungen von Maßnahmen sind nicht annähernd erschöpfend untersucht; die Analysen der Interaktion von Instrumenten muss weiterentwickelt und Wirkungsforschung muss besser mit Implementationsforschung verknüpft werden; die Evaluation der vertikalen wie horizontalen Koordination von Institutionen und Akteuren steckt noch in den Kinderschuhen; nach wie vor fehlt es an robusten Analysen der Makrowirkung von Arbeitsmarktpolitik. In der Evaluation der Arbeitsmarktpolitik und im Prozess der daran ausgerichteten Reformen verhält es sich wie beim Schwimmen gegen den Strom: Eine Verminderung der Anstrengungen hieße, zurückgerissen zu werden.

Günther Schmid, Direktor der Abteilung Arbeitsmarktpolitik und Beschäftigung am Wissenschaftszentrum Berlin für Sozialforschung (WZB) und Professor für Ökonomische Theorie der Politik an der Freien Universität Berlin. e-mail: gues@wzb.eu 